02

\title{
Наблюдение когерентного пленения населенности в ансамблях NV-центров в алмазе в условиях антипересечения уровней основного состояния
}

\author{
(C) Р.А. Ахмеджанов ${ }^{1,2}$, И.В. Зеленский ${ }^{1,2}$, Л.А. Гущин ${ }^{1,2}$, В.А. Низов ${ }^{1,2}$, Н.А. Низов ${ }^{1,2}$, Д.А. Собгайда ${ }^{1,2}$ \\ ${ }^{1}$ Институт прикладной физики РАН, \\ 603950 Нижний Новгород, Россия \\ ${ }^{2}$ Казанский фризико-технический институт им. Завойского, \\ 420029 Казань, Россия \\ e-mail: rinat@appl.sci-nnov.ru
}

Поступила в редакцию 15.02.2019г.

В окончательной редакции 15.02.2019г.

Принята к публикации 15.03.2019г.

Реализован эффект когерентного пленения населенности в ансамблях NV-центров в алмазе в условиях антипересечения уровней основного состояния при наложении сильного внешнего магнитного поля, направленного вдоль оси [111] кристалла.

Ключевые слова: двухфотонная отстройка, кросс-релаксация, резонанс.

DOI: $10.21883 /$ OS.2019.08.48038.67-19

\section{Введение}

Центры окраски в алмазе вызывают широкий интерес благодаря уникальным особенностям алмаза, таким как оптическая прозрачность, слабое электрон-фононное взаимодействие, высокая стабильность оптически активных центров, в том числе при комнатной температуре. Одним из перспективных считается NV-центр из-за возможного его применения для наноразмерного зондирования магнитных и электрических полей [1], измерения локальной температуры [2], реализации квантовых вычислений [3], а также стабильных флуоресцентных биомаркеров [4]. Для многих приложений, связанных с использованием датчиков и кубитов на основе центров окраски, применение квантовых интерференционных эффектов: когерентного пленения населенности (КПН) или электромагнитно индуцированной прозрачности (ЭИП) является преимущественным, поскольку эти эффекты не требуют СВЧ возбуждения для создания или манипулирования спиновой когерентностью. Еще одним преимуществом КПН/ЭИП является то, что центры окраски, которые демонстрируют отсутствие или плохой контраст при использовании оптически детектируемого магнитного резонанса для считывания спиновых состояний, потенциально могут иметь высокий контраст при использовании КПН/ЭИП.

Эффект электромагнитно индуцированной прозрачности в ансамблях NV-центров в алмазе реализован на сегодняшний день в условиях антипересечения уровней основного состояния при наложении сильного внешнего магнитного поля [5] и при квазинулевых магнитных полях при внутренних или внешних напряжениях, соответствующих антипересечению уровней возбужденного состояния [6]. Эксперименты в геометрии когерентного пленения населенности проводились в основном при малых магнитных полях $[7,8]$ и преимущественно с одиночными центрами. В настоящей работе представлены результаты экспериментального наблюдения эффекта когерентного пленения населенности в ансамбле азот-вакансионных центров в условиях антипересечения уровней основного состояния.

\section{Эксперимент}

В наших исследованиях использовался образец синтетического алмаза (Element Six), облученный электронным пучком (доза облучения $10^{18}$ электронов $/ \mathrm{cm}^{2}$ ) и затем отожженный при температуре $800^{\circ} \mathrm{C}$.

Для реализации интерференционных эффектов при малых магнитных полях требуется $\Lambda$-схема уровней, образующаяся при антипересечении уровней возбужденного состояния в результате действия внутренних напряжений в кристалле алмаза $[9,10]$. Требуемые для таких схем напряжения достаточно малы и соответствуют неоднородному уширению бесфононной линии около $10-15 \mathrm{GHz}$. В используемом нами образце ширина бесфононной линии порядка $750 \mathrm{GHz}$, следовательно, число „полезных“ центров, в которых реализуется $\Lambda$-схема, очень мало. Поэтому в наших экспериментах по реализации эффекта когерентного пленения населенности эффективная $\Lambda$-схема создавалась за счет достаточно сильного (порядка 0.1 Т) магнитного поля, наложенного вдоль направления [111] кристалла. Известно, что при наложении сильного магнитного поля (в условиях антипересечения уровней основного состояния) эффективность когерентных эффектов существенным образом зависит от того, насколько хорошо ось кристалла ориентирована относительно внешнего поля [5]. 
Известны методы контроля качества ориентировки: по ширине провала в люминесценции в окрестности точки антипересечения основного состояния [11], по времени декогеренции [12], по профилю оптически детектируемого магнитного резонанса в присутствии магнитного поля [13], по форме провала в люминесценции NV-центров при поле $590 \mathrm{G}$ [14]. Однако все эти методы либо не дают необходимой точности, либо слишком трудоемки. Поэтому мы разработали простую оригинальную методику определения угла между осью кристалла и направлением магнитного поля. Суть ее состоит в следующем. Мы использовали измерение положений кроссрелаксационных резонансов при сканировании магнитного поля катушкой в присутствии дополнительного постоянного магнитного поля (см. подробнее [15]). Сканируемое магнитное поле за счет эффекта Зеемана изменяет частоты микроволновых переходов NV-центров. Кросс-релаксация наблюдается при совпадении частот микроволновых переходов различных по направлению групп NV-центров в алмазе, в результате чего в сигнале флуоресценции наблюдается провал.

В общем случае положения резонансов описываются формулой

$$
B_{i}=-\frac{\left(\mathbf{B}^{D C}, \boldsymbol{v}_{i}\right)}{\left(\mathbf{b}^{\text {scan }}, \boldsymbol{v}_{i}\right)},
$$

где $\mathbf{B}^{D C}-$ постоянное магнитное поле, $\mathbf{b}^{\text {scan }}-$ единичный вектор, задающий направление сканирования магнитного поля, векторы $\boldsymbol{v}_{i}=\mathbf{e}_{k} \pm \mathbf{e}_{i}$ являются комбинациями векторов $\mathbf{e}_{i}=[1, \pm 1, \pm 1]$, описывающих возможные ориентации NV-центров в кристаллической решетке. Приложение постоянного магнитного поля $\mathbf{B}^{D C}$, имеющего компоненту поперек направления $\mathbf{b}^{\text {scan }}$, необходимо для расщепления кросс-релаксационных резонансов (если $\mathbf{B}^{D C} \| \mathbf{b}^{\text {scan }}$, то каждый из резонансов смещается одинаково).

Для описания результатов удобно воспользоваться системой координат, связанной с кристаллографическими осями алмаза $x=[100], y=[010], z=[001]$. В случае, когда угол $\theta$ между сканируемым полем и направлением [111] мал $\left(\right.$ т.е. $\left.\mathbf{b}^{\text {scan }} \simeq\left(\frac{1}{\sqrt{3}}, \frac{1}{\sqrt{3}}, \frac{1}{\sqrt{3}}\right)\right)$, в магнитных полях меньше $100 \mathrm{G}$ могут наблюдаться до 6 резонансов:

$$
\begin{gathered}
B_{[100]}=-\frac{B_{x}^{D C}}{b_{x}^{\text {scan }},} \\
B_{[010]}=-\frac{B_{y}^{D C}}{b_{y}^{\text {scan }}}, \\
B_{[001]}=-\frac{B_{z}^{D C}}{b_{z}^{s c a n}}, \\
B_{[110]}=-\frac{B_{x}^{D C}+B_{y}^{D C}}{b_{x}^{\text {scan }}+b_{y}^{\text {scan }}}, \\
B_{[101]}=-\frac{B_{x}^{D C}+B_{z}^{D C}}{b_{x}^{\text {scan }}+b_{z}^{\text {scan }}},
\end{gathered}
$$

$$
B_{[011]}=-\frac{B_{y}^{D C}+B_{z}^{D C}}{b_{y}^{s c a n}+b_{z}^{s c a n}} .
$$

В ходе эксперимента подбиралось постоянное магнитное поле $\mathbf{B}^{D C}$, при котором наблюдались шесть отщепленных резонансов (см., например, рис. 1), положения которых входят в левые части уравнений системы (1). Для увеличения точности измерений использовались все положения резонансов и решалась переопределенная система. При этом использовался метод наименьших квадратов. Мы искали минимум функции $F$ пяти переменных - трех проекций $\mathbf{B}^{D C}$ и двух переменных, задающих направление сканирования магнитного поля (например, $b_{x}^{s c a n}$ и $\left.b_{y}^{s c a n}\right)$. Функция $F$ описывала отличие измеренных положений резонансов от рассчитанных для данного набора переменных. Для значений проекций $\mathbf{B}^{D C}$ и направления сканирования магнитного поля, при котором достигался минимум $F$, мы вычисляли угол $\theta$. Для совмещения направления магнитного поля и оси [111] мы изменяли положение образца в катушке и проводили измерение угла $\theta$, добиваясь уменьшения его значения.

Схема экспериментальной установки для наблюдения КПН представлена на рис. 2. Излучение одночастотного лазера на красителе (выходная мощность $150 \mathrm{~mW}$, ширина линии $1 \mathrm{MHz}$, длина волны $637 \mathrm{~nm}$ ) расщеплялось на два пучка при помощи поляризационного делителя. Соотношение интенсивностей в пучках контролировалось полуволновой пластиной, расположенной перед делителем. Для создания необходимой частотной отстройки между управляющим и пробным излучением пучки направлялись на два акустооптических модулятора, включенных один в двухпроходовой, а другой в однопроходовой схеме. Акустооптические модуляторы обеспечивали двухчастотную отстройку в пределах от 0 до $200 \mathrm{MHz}$. После прохождения акустооптических модуляторов пучки совмещались на поляризационном делителе и фокусировались на кристалле, помещенном в сверхпроводящий соленоид, создающий магнитное

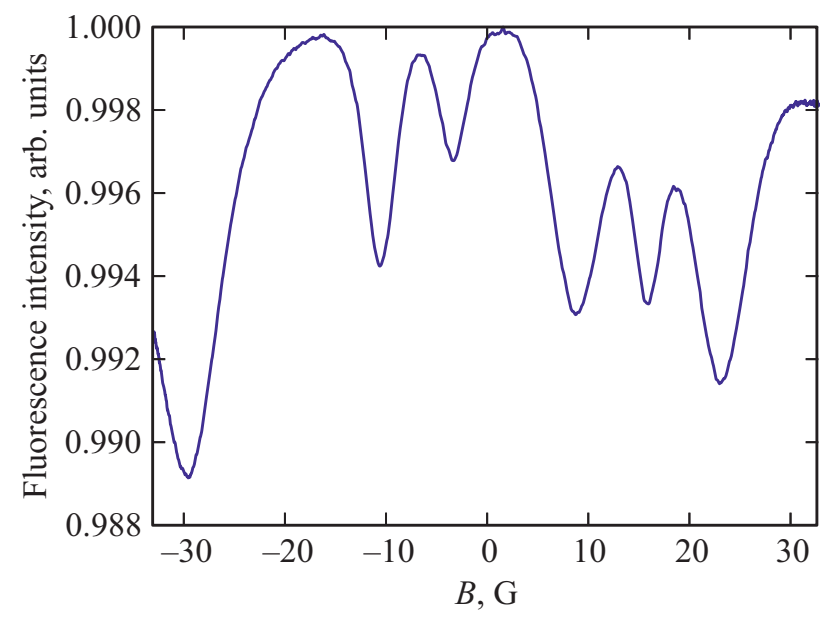

Рис. 1. Зависимость интенсивности флуоресценции от величины сканируемого поля при наличии постоянного поля. 


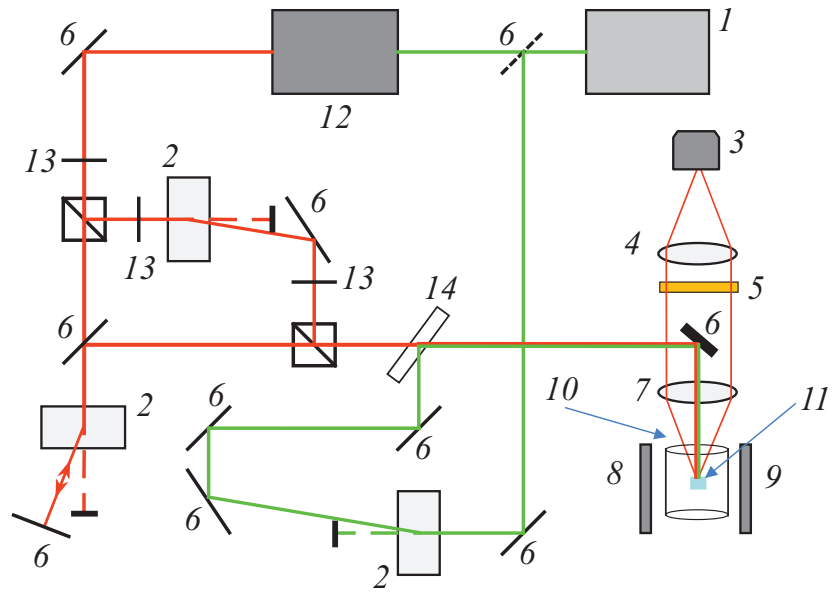

Рис. 2. Схема установки: 1 - лазер накачки, 2 - АОМ, 3 - фотодиод, 4, 7 - линзы, 5 - светофильтр, 6 - зеркало, 8,9 - магнитные катушки, 10 - соленоид, 11 - образец, 12 - лазер на красителе, 13 - пластинка $\lambda / 2,14-$ дихроическое зеркало.

поле (кристалл предварительно был ориентирован при комнатной температуре по методике, описанной выше). Кристалл вместе с соленоидом погружались в гелиевый криостат (рабочая температура $2 \mathrm{~K}$ ). Длина волны лазерного излучения настраивалась на центр бесфононной линии в спектре возбуждения, что обеспечивало максимальное значение сигнала флуоресценции. Заметим, что центры бесфононной линии в спектре возбуждения и люминесценции отличались. Этот факт был отмечен в работе [16], однако для нашего кристалла при температуре $2 \mathrm{~K}$ сдвиг существенно больше $(300 \mathrm{GHz})$, чем для образца, исследованного в работе [17] (порядка $20 \mathrm{GHz}$ ). Флуоресценция детектировалась на длинах волн более $650 \mathrm{~nm}$. Дополнительно к этому часть излучения лазера накачки $(532 \mathrm{~nm})$ также направлялась на образец, иначе большая часть NV-центров не будет взаимодействовать с резонансным излучением из-за выжигания спектрального провала и ионизации спустя очень короткое время [5]. Для изменения угла между осью [111] и магнитным полем в ходе эксперимента использовалась пара катушек, размещенных вне криостата.

Для измерения зависимости флуоресценции от двухфотонной отстройки использовалась схема детектирования на основе синхронного усилителя. Помимо медленного сканирования, частота пробного луча, а следовательно, и двухфотонная отстройка дополнительно модулировались при помощи низкочастотного генератора сигналов. Сигнал с синхровыхода генератора затем подавался на вход синхронного усилителя, который выделял и усиливал сигнал флуоресценции с фотодиода только на частоте модуляции. Частота модуляции $(50 \mathrm{kHz})$ выбиралась отличной от ожидаемых частот экспериментальных шумов, но достаточно малой по сравнению с ожидаемой скоростью установления эффекта КПН. Полный размах модуляции двухфотонной отстройки выбирался сравнимым с ожидаемой полушириной провала
КПН (5 MНz). Как показали модельные эксперименты, использование такой схемы позволяет эффективно детектировать провалы, контраст которых составляет доли процента.

\section{Результаты и обсуждение}

На рис. 3 представлена характерная зависимость выходного сигнала синхронного усилителя в зависимости от частотной отстройки. Отчетливо виден резонанс когерентного пленения населенности. Ширина резонансов порядка 8-10 MHz. Контраст резонансов очень мал (по нашим оценкам не превышает долей процента). Одной из причин низкого контраста может быть следующее. Наши эксперименты по выжиганию долгоживущих спектральных провалов показали, что в используемом кристалле ширина спектрального провала порядка $200 \mathrm{MHz}$ (возможно, за счет спектральной диффузии, особенно важной при низких температурах). Таким образом, имеется сильное спектральное перекрытие каналов возбуждения на смежных переходах $\Lambda$-схемы (двухфотонная отстройка порядка нескольких десятков $\mathrm{MHz}$ ), что сильно нивелирует интерференцию каналов возбуждения, приводя к существенному уменьшению контраста когерентного пленения населенности. Увеличение двухфотонной отстройки за счет изменения магнитного поля (ухода от точки антипересечения) или угла между магнитным полем и осью кристалла нежелательно, так как при этом существенно ухудшаются параметры $\Lambda$-схемы. Действительно, контраст наблюдаемых нами резонансов падает при смещении из точки антипересечения уровней основного состояния и при увеличении угла между осью кристалла и направлением магнитного поля (изменялось при помощи катушек, расположенных вне криостата). В этом смысле мы находимся в гораздо худших условиях, чем в экспериментах [5], где двухфотонная отстройка значительно превышала ширину линии и был достигнут контраст $17 \%$ при наблюдении электромагнитно индуцированной прозрачности.

На рис. 4 показано местоположение резонансов (двухфотонной отстройки) в зависимости от внешнего магнитного поля. Пользуясь этими данными, можно определить угол $\theta$ между магнитным полем и направлением [111]. Известно, что для группы NV-центров, ориен-

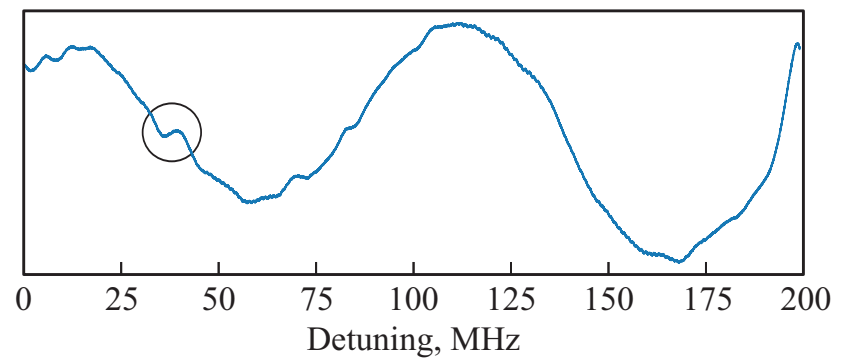

Рис. 3. Зависимость выходного сигнала синхронного усилителя в зависимости от частотной отстройки при значении приложенного магнитного поля $1039 \mathrm{G}$. 


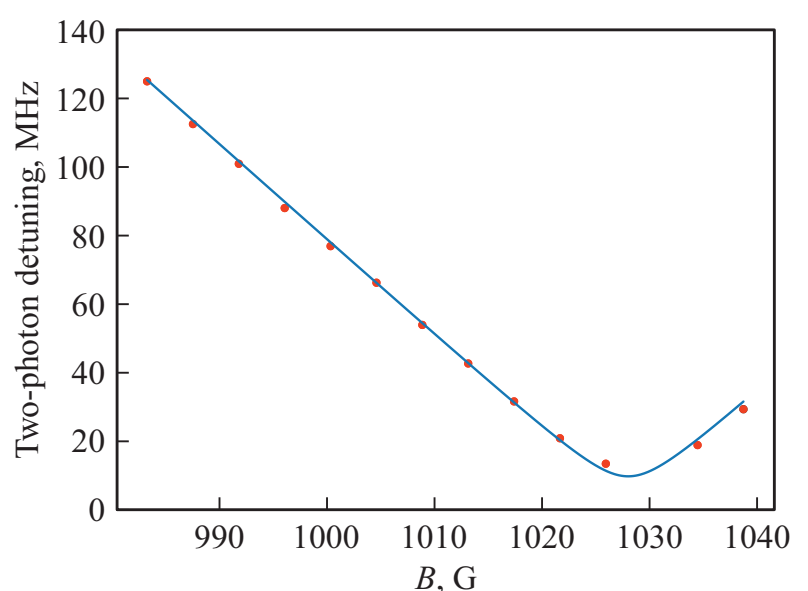

Рис. 4. Местоположение резонансов (двухфотонной отстройки) в зависимости от внешнего магнитного поля (точки). Сплошная линия - результат расчета. $\theta=0.14^{\circ}$.

тированной вдоль [111], частота перехода $|0\rangle \leftrightarrow|-1\rangle f$ (а следовательно, и двухфотонная отстройка) зависит от величины и направления магнитного поля следующим образом [13]:

$$
f=\frac{2 \sqrt{3} D}{3}\left(3 b^{2}+1\right)^{\frac{1}{2}} \sin (\alpha / 3+2 \pi / 3),
$$

где $b=g \mu_{B} B / D-$ безразмерное магнитное поле, $D \approx 2.88 \mathrm{GHz}$, параметр $\alpha$ определяется выражением $\cos \alpha=\frac{9 b^{2}-27 b^{2} \cos ^{2} \theta+2}{2\left(3 b^{2}+1\right)^{\frac{3}{2}}}$.

Варьируя $\theta$, мы обнаружили, что максимальное совпадение этой расчетной зависимости с экспериментом наблюдается при угле $\theta=0.14^{\circ}$ (сплошная линия на рис. 4). Это показывает, что наш метод определения угла на основе кросс-релаксационных резонансов действительно позволил добиться направления магнитного поля, близкого к оси [111], что говорит о его эффективности.

\section{Заключение}

В данной работе приведены результаты экспериментов по наблюдению эффекта когерентного пленения населенности в ансамблях NV-центров в алмазе в условиях антипересечения уровней основного состояния. Разработан и успешно апробирован метод контроля угла между осью кристалла и направлением магнитного поля на основе кросс-релаксационных резонансов. Полученные результаты являются еще одним экспериментальным подтверждением возможности формирования суперпозиционных состояний в ансамблях NV-центров в алмазе и полностью оптического контроля спиновых состояний без использования микроволнового излучения.

\section{Благодарности}

Авторы благодарны Ф.Р. Хеммеру (Техасский университет A\&M) за полезные обсуждения.

\section{Финансирование работы}

Работа выполнена в рамках гранта Правительства РФ по постановлению 220, договор с ведущей организацией КФТИ ФИЦ КазНЦ РАН № 14.W03.31.0028, а также госконтракта с ФИЦ ИПФ РАН, проект № 0035-2019-0004 (в части, касающейся методики ориентации кристалла относительно внешнего магнитного поля).

\section{Конфликт интересов}

Авторы заявляют, что у них нет конфликта интересов.

\section{Список литературы}

[1] Taylor J.M., Cappellaro P., Childress L., Jiang L., Budker D., Hemmer P.R., Yacoby A., Walsworth R., Lukin M.D. // Nature Physics. 2008. V. 4. P. 810.

[2] Kucsko G., Maurer P.C., Yao N.Y., Kubo M., Noh H.J., Lo P.K., Park H., Lukin M.D. // Nature. 2013. V. 500. P. 54.

[3] Gurudev Dutt M.V., Childress L., Jiang L., Togan E., Maze J., Jelezko F., Zibrov A.S., Hemmer P.R., Lukin M.D. // Science. 2007. V. 316. P. 1312.

[4] Alkahtani M.H., Alghannam F., Jiang L., Almethen A., Rampersaud A.A., Brick R., Gomes C.L., Scully M.O., Hemmer P.R. // Nanophotonics. 2018. V. 7. P. 1423-1453.

[5] Hemmer P.R., Turukhin A.V., Shahriar M.S., Musser J.A. // Opt. Lett. 2001. V. 26. P. 361.

[6] Acosta V.M., Jensen V.M., Santori C., Budker D., Beausoleil R.G. // Phys. Rev. Lett. 2013. V. 110. P. 213605.

[7] Santori C., Tamarat P., Neumann P., Wrachtrup J., Fattal D., Beausoleil R.G., Rabeau J., Olivero P., Greentree A.D., Prawer S., Jelezko F., Hemmer P. // Phys. Rev. Lett. 2006. V. 97. P. 247401.

[8] Golter D.A., Dinyari K.N., Wang H. // Phys. Rev. A. 2013. V. 87. P. 035801.

[9] Tamarat Ph., Manson N.B., Harrison J.P., McMurtrie R.L., Nizovtsev A., Santori C., Beausoleil R.G., Neumann P., Gaebe T., Jelezko F., Hemmer P., Wrachtrup J. // New J. Phys. 2008. V. 10. P. 045004.

[10] Doherty M.W., Manson N.B., Delaney P., Hollenberg L.C.L. // New J. Phys. 2011. V. 13. P. 025019.

[11] Epstein R.J., Mendoza F.M., Kato Y.K., Awschalom D.D. // Nature Physics. 2005. V. 1. P. 94-98.

[12] Stanwix P.L., Pham L.M., Maze J.R., Le Sage D., Yeung T.K., Cappellaro P., Hemmer P.R., Yacoby A., Lukin M.D., Walsworth R.L. // Phys. Rev. B. 2010. V. 82. P. 201201.

[13] Doherty M.W., Michl J., Dolde F., Jakobi I., Neumann P., Manson N.B., Wrachtrup J. // New J. Phys. 2014. V. 16. P. 063067.

[14] Anishchik S.V., Vins V.G., Yelisseyev A.P., Lukzen N.N., Lavrik N.L., Bagryansky V.A. // New J. Phys. 2015. V. 17. P. 023040.

[15] Akhmedzhanov R., Gushchin L., Nizov N., Nizov V., Sobgayda D., Zelensky I., Hemmer P. // Phys. Rev. A. 2017. V. 96. P. 013806.

[16] Kiisk V., Palm V., Suisalu A., Sildos I. // J. Luminesc. 2000. V. 86. P. 349-353.

[17] Holliday K., Manson N.B., Glasbeek M., van Oort E. // J. Phys.: Condens. Matter. 1989. V. 1. P. 7093. 